\title{
Endoscopic ultrasonography of solitary rectal ulcer syndrome
}

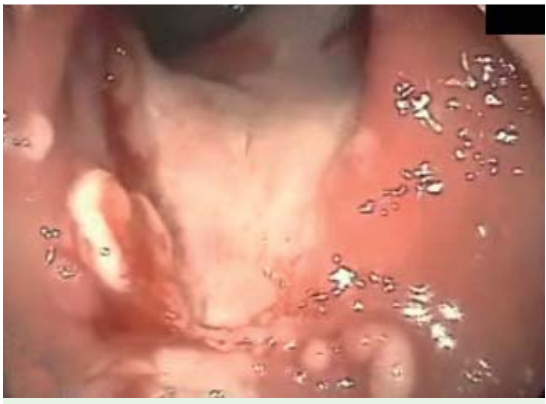

Fig. 1 Sigmoidoscopic view showing a large ulcer in the posterior rectal mucosa.

Solitary rectal ulcer syndrome (SRUS) is an uncommon poorly understood benign disorder of defecation. SRUS may simulate other disorders such as inflammatory bowel disease and neoplasms. Histopathological analysis forms the cornerstone of diagnosis for SRUS.Key histological features include fibromuscular obliteration of the lamina propria with splaying of the muscularis mucosae upward between the crypts, thickened mucosa, and glandular distortion [1].

Endoscopic ultrasonography (EUS) can be useful in the evaluation of SRUS. The characteristic findings are thick hyperechoic submucosa and thick hypoechoic muscularis propria with an intermediate hyperechoic layer. Linear EUS imaging shows a transition zone where the first interface layer and the muscularis mucosae disappear, and the submucosa gradually becomes thicker. The presence of an interface between the two muscular layers and between the muscular layer and the submucosa rules out malignant infiltration [2]. The hyperechoic band in the muscularis propria in SRUS has been attributed to a fibrous septum while the hyperechoic submucosa is due to a fibrotic lamina propria [3].

A 65-year-old man presented with a history of bleeding per rectum and straining at stool for 6 months. Sigmoidoscopy showed a large ulcer in the posterior rectal mucosa approximately $8 \mathrm{~cm}$ above the anal verge ( $\bullet$ Fig. 1; Video 1 ). Biopsies were sent for histopathological examination and a linear EUS was performed. Linear EUS of the anal canal revealed normal thickness of the anal sphincters, but there was thickening of the submucosa as

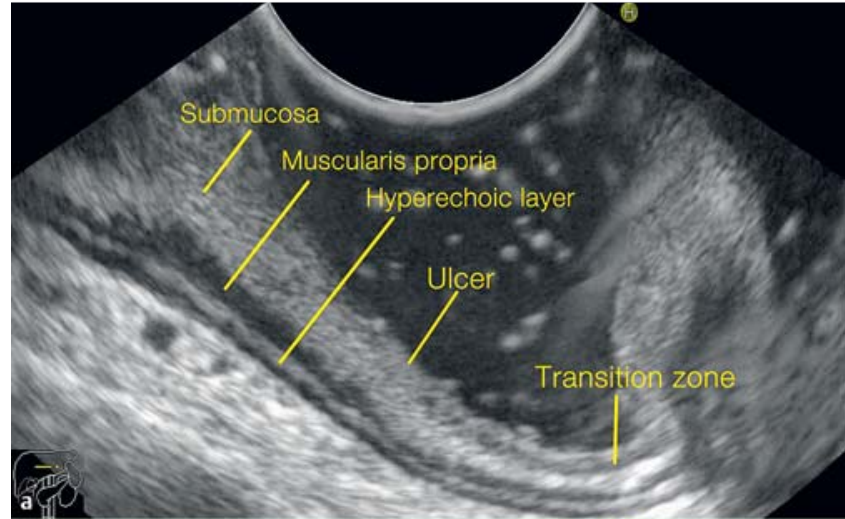

Fig. 2 Linear endoscopic ultrasonography (EUS) images showing: a thickening of the submucosa and muscularis propria; $\mathbf{b}, \mathbf{c}$ the solitary rectal ulcer in an otherwise normal rectum and the transition zone; d a hyperechoic layer in the thickened hypoechoic muscularis propria.
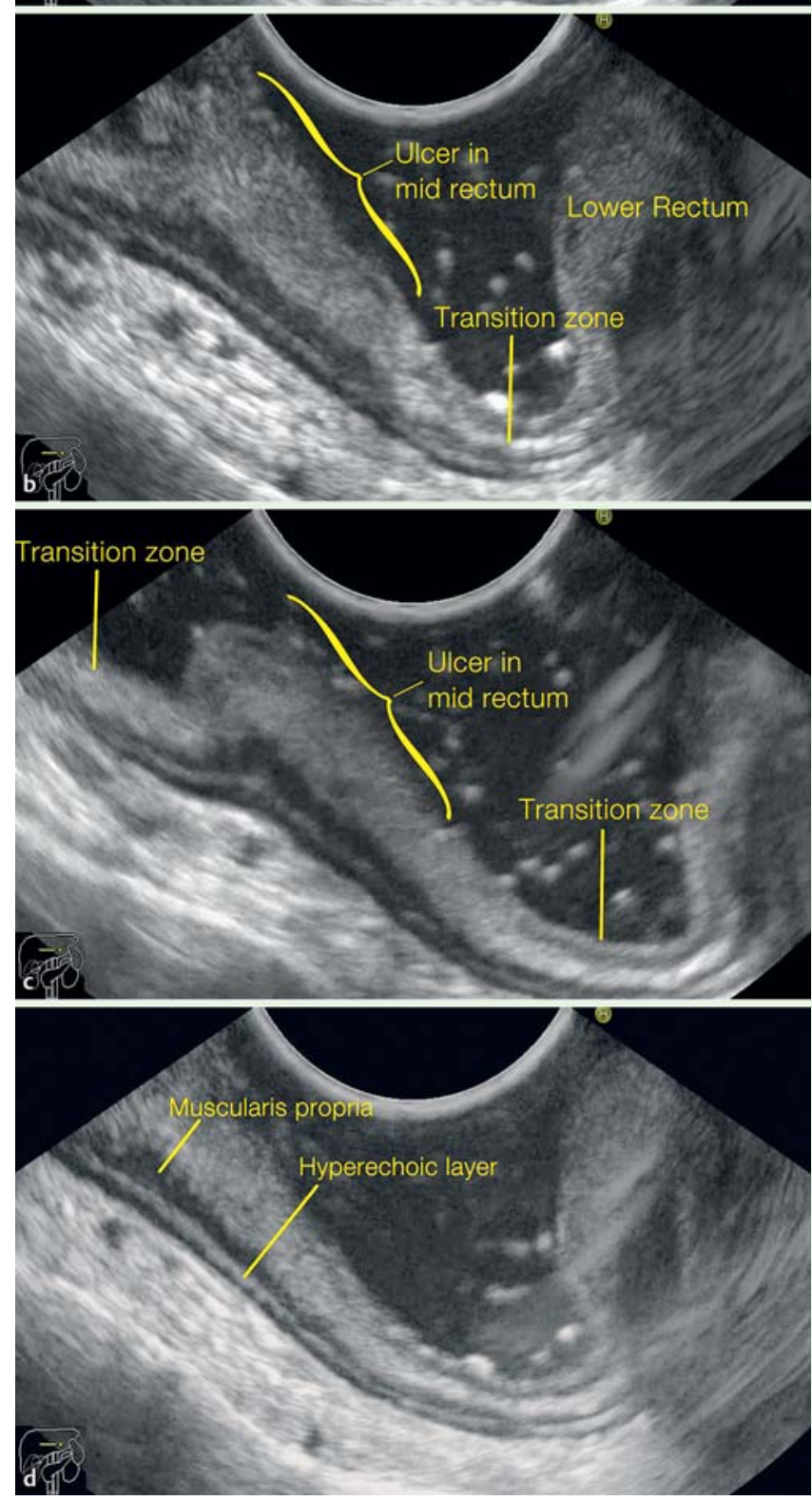


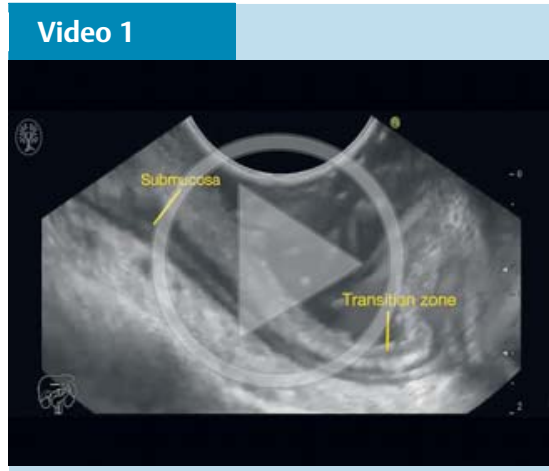

Sigmoidoscopy showed a large ulcer in the posterior rectal mucosa approximately $8 \mathrm{~cm}$ above the anal verge, suggestive of solitary rectal ulcer syndrome (SRUS). Linear endoscopic ultrasonography (EUS) of the rectum revealed: a solitary rectal ulcer in an otherwise normal rectum as well as the transition zone; thickening of the submucosa and muscularis propria, with a hyperechoic layer in the thickened hypoechoic muscularis propria.

well as the muscularis propria ( $\bullet$ Fig. 2a). A transition zone was visible where the first interface layer and muscularis mucosae disappeared and the submucosa gradually became thicker ( $\bullet$ Figs. $\mathbf{2 b}, \mathbf{c}$; - Video 1). The plane between the submucosa and muscularis propria could be clearly identified. Deep to the submucosa, the muscularis propria was thickened and was split by a hyperechoic band around the entire circumference, which signified a plane of normal tissue between the thickened circular and longitudinal muscles of the muscularis propria ( $\boldsymbol{\bullet}$ Fig. $\mathbf{2 d}$ ). The diagnosis of SRUS was confirmed by histopathology ( $\bullet$ Fig. 3 ). The patient was reassured that his condition did not represent malignancy and he underwent biofeedback.

The use of rectal ultrasonography was helpful in ruling out an associated malignancy in this patient and should be routinely done as a part of the evaluation in cases of suspected SRUS.

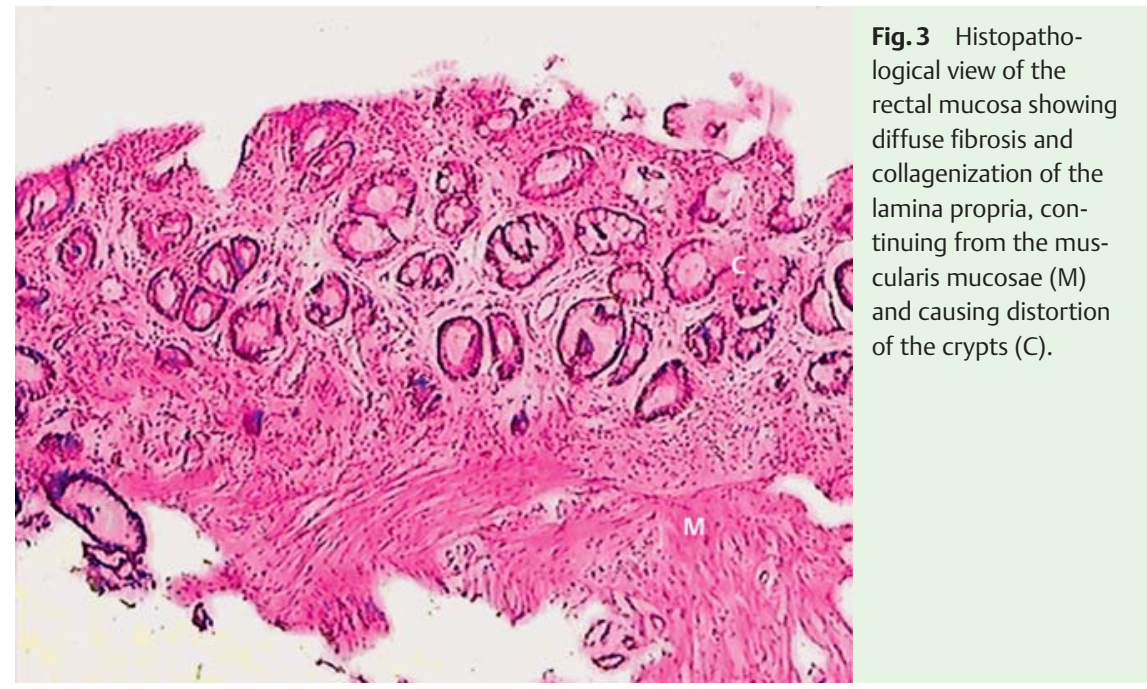

Endoscopy_UCTN_Code_CCL_1AF_2AH

Competing interests: None

\section{Malay Sharma ${ }^{1}$, Piyush Somani', Amol Patil', Avinash Kumar', Charu Shastri' ${ }^{2}$}

${ }^{1}$ Department of Gastroenterology, Jaswant Rai Speciality Hospital, Meerut, Uttar Pradesh, India

${ }^{2}$ Department of Pathology, Jaswant Rai Speciality Hospital, Meerut, Uttar Pradesh, India

\section{References}

1 Abid S, Khawaja A, Bhimani SA et al. The clinical, endoscopic and histological spectrum of the solitary rectal ulcer syndrome: a single-center experience of 116 cases. BMC Gastroenterol 2012; 14: 72

2 Sharma M, Somasundaram A. Endoscopic ultrasonography for an ulcer in the rectum. Gastroenterology 2011; 141: e7-e8

3 Petritsch W, Hinterleitner TA, Aichbichler B et al. Endosonography in colitis cystica profunda and solitary rectal ulcer syndrome. Gastrointest Endosc 1996; 44: 746 - 751
Bibliography

Dol http://dx.doi.org/

10.1055/s-0042-102449

Endoscopy 2016; 48: E76-E77

(c) Georg Thieme Verlag KG

Stuttgart · New York

ISSN 0013-726X

Corresponding author

Malay Sharma, MD, DM

Saket

Meerut

Uttar Pradesh - 250001

India

Fax: +91-121-2657154

sharmamalay@hotmail.com
Jaswant Rai Speciality Hospital 